\title{
Implementation of Multi-hop Cluster based Routing Protocol for Wireless Sensor Networks
}

\author{
S.Koteswararao \\ Associate Professor \\ Sri Mittapalli college of \\ Engineering, \\ Tummalapalem,Guntur(Dt), \\ AP,India
}

\author{
M.Sailaja \\ Professor ECE \\ UCE,Kakinada,JNTUK \\ Kakinada,E.G.Godavari(Dt)
}

\author{
T.Madhu \\ Principal\& Professor \\ SIET,Narsapur \\ West Godavari(Dt)
}

\begin{abstract}
Clustering routing protocol provides an effective method for prolonging the lifetime of a wireless sensor network. But most of the researches care less about the communication between Cluster Head (CH) nodes [4] and Base Station (BS). This paper proposed a Multi-hop Cluster based Routing Protocol for long range transmission in wireless sensor network. The protocol consists of two parts, one is with respect to cluster management in sensor area, and the other is about the data transmission between base station and the sensor area. Simulation results show that the protocol offers a better performance than single-hop clustering routing protocols in terms of network lifetime and energy consumption when the base station is located far away from the sensing area.
\end{abstract}

Keywords - Wireless Sensor Networks, Gateway Node, Energy Efficient, Cluster head, Multi-hop Clustering.

\section{INTRODUCTION}

Wireless Sensor Networks (WSNs) are formed by hundreds or thousands of nodes that gather information and forward it to a sink node. Distinguished from traditional wireless networks, sensor networks are characterized of severe power computation, and memory constraints [1]. As the wireless nodes in a WSN are usually driven by power sources (e.g. batteries) which are irreplaceable, energy resource of sensor networks should be managed wisely to extend the lifetime of sensors. Routing protocol is one of the core technologies in the WSN. Due to its inherent characteristics, routing is full of challenge in WSN [2]. Clustering is a well-know and widely used exploratory data analysis technique, and it is particularly useful for applications that require scalability to hundreds or thousands of nodes [3]. For large-scale networks, node clustering has been proposed for efficient organization of the sensor network topology, and prolonging the network lifetime.

We consider a network of energy-constrained sensors that are deployed over a geographic area for monitoring the environment. Among the sources of energy consumption in a sensor node, wireless data transmission is the most critical. Within a clustering organization, intra-cluster communication can be single hop or multi-hop, as well as inter-cluster communication[12]. Low Energy Adaptive Clustering Hierarchy $(\mathrm{LEACH})$ is the first clustering protocol that was proposed for reducing power consumption [4]. It forms clusters by using a distributed algorithm, each node has a certain probability of becoming a cluster head per round, and the task of being a cluster head is rotated between nodes. A non- $\mathrm{CH}$ node determines its cluster by choosing the $\mathrm{CH}$ that can be reached with the least communication energy consumption. In the data transmission stage, each cluster head sends an aggregated packet to the base station by single hop. Hybrid Energy-Efficient Distributed (HEED) clustering approach is one of the most recognized energy-efficient clustering protocols [5]. It extends the basic scheme of LEACH by using residual energy and node degree or density. In HEED, the initial probability for each node to become a tentative cluster head depends on its residual energy, and final heads are selected according to the intra-cluster communication cost. The clustering process is divided into a number of iterations, and terminates within a constant number of iterations. HEED achieves fairly uniform distribution of cluster heads across the network consumption. In the data transmission stage, each cluster head sends an aggregated packet to the base station by single hop. Hybrid Energy-Efficient Distributed (HEED) clustering approach is one of the most recognized energyefficient clustering protocols. It extends the basic scheme of LEACH by using residual energy and node degree or density. In HEED, the initial probability for each node to become a tentative cluster head depends on its residual energy, and final heads are selected according to the intra-cluster communication cost. The clustering process is divided into a number of iterations, and terminates within a constant number of iterations[7]. HEED achieves fairly uniform distribution of cluster heads across the network. Although the protocols above reduce energy consumption on forwarding paths to increase energy efficiency, but they all use one-hop communication between $\mathrm{CH}$ nodes and base station. Notice that the base station is usually located far away from the sensing area. In this case, each $\mathrm{CH}$ nodes forward the data to the base station directly probably consumes much more energy[8], and it does not necessarily balance the energy consumption among the networks. Thus recent researches let cluster heads cooperate with each other to forward their data to the base station. Energy-Efficient Unequal Clustering (EEUC) wisely organizes the network via unequal clustering and multi-hop routing. In EEUC [13], cluster heads are elected by localized competition, which is unlike LEACH, and with no iteration, which differs from HEED. And the node's competition range. Although the protocols above reduce energy consumption on forwarding paths to increase energy efficiency, but they all use one-hop communication between $\mathrm{CH}$ nodes and base station. Notice that the base station is usually located far away from the sensing area. In this case, each $\mathrm{CH}$ nodes forward the data to the base station directly probably consumes much more energy, and it does not necessarily balance the energy consumption among the networks. Thus recent researches let 
cluster heads cooperate with each other to forward their data to the base station. Energy-Efficient Unequal Clustering (EEUC) [6] wisely organizes the network via unequal clustering and multi-hop routing. In EEUC, cluster heads are elected by localized competition, which is unlike LEACH, and with no iteration, which differs from HEED. And the node's competition range decreases as its distance to the base station decreasing. By using this unequal clustering pattern, EEUC successfully balances the energy consumption over the network. But when the sensor area is far away from the base station, problem of unbalanced energy consumption among cluster heads still exists. As the cluster heads closer to the base station are burdened with heavier relay traffic, and they may drain their energy and die much faster than other nodes in the networks. Many researches dedicate to energy efficient routing of WSNs [7-10], but most of them care less about the communication between Cluster Head $(\mathrm{CH})$ nodes and Base Station (BS). In this paper, we propose and evaluate a Multihop Clustering Routing Protocol (MCR) for mitigating the existent problem. MCR consists of two parts, one is with respect to cluster management in sensor area, and the other is about the data transmission between base station and the sensor area. The remainder of this paper is organized as follows. Section 2 introduces a single-hop clustering routing protocol, HEED. Section 3 describes the MCR protocol. Section 3 contains performance evaluation of our scheme throughout simulations. Finally, we conclude the paper in section 4.

\section{SINGLE HOP CLUSTERING ROUTING PROTOCOL}

Employ clustering techniques in routing protocols can hierarchically organizing network topology and to prolong the lifetime of a wireless sensor network. A single-hop clustering routing protocol can reduce the communication overhead by selecting a $\mathrm{CH}$ to forward data to base station via one hop [4]. As shown in Fig. 1, the transmission range will be decreased obviously with clustering. Many single hop clustering routing pr otocol have been proposed like LEACH, HEED as discussed in section1.
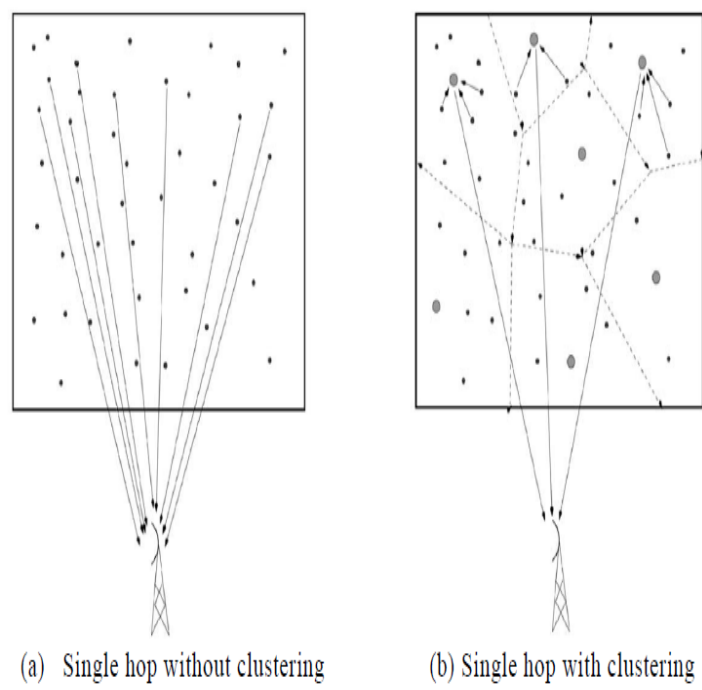

(b) Single hop with clustering

Fig. 1 Clustering Routing Protocol

HEED protocol is a distributed single-hop clustering routing protocol for Wireless Sensor Networks, which selecting CHs based on both residual energy of each node and communication cost. It has the advantages in increasing the network lifetime and having a constant algorithm complexity [14]. HEED uses two clustering parameter to select $\mathrm{CH}$ : one is residual energy, and the other is communication cost. The communication cost is defined as an average minimum reach ability power, which means the minimum power levels required by all nodes within the cluster range to reach the $\mathrm{CH}$. The communication cost held by $\mathrm{CHs}$ is used to let a node which belong to several $\mathrm{CH}$ s choose the best one. In HEED, each node must be mapped to exactly one cluster, and each node belongs to its only $\mathrm{CH}$ within one hop. After a clustering process, each node can either elect to become a $\mathrm{CH}$ due to a probability or join a cluster according to $\mathrm{CH}$ messages $[13,14]$.

\section{MULTI HOP CLUSTERING ROUTING PROTOCOL}

To increases the network lifetime and well balances the energy consumption, we adopt an energy driven method to rotate cluster-head, and propose a Multi-hop Clustering Routing Protocol (MCR) for long range transmission in the wireless sensor networks.

\subsection{System Model}

This protocol mainly includes three stages: Build up the network topology, Data collection and transmission and Cluster-head rotation and network topology rebuild. We make some assumptions about the sensor nodes and the underlying network model:

3.1.1 There is a base station (i.e. data sink) located far away from the sensing field. Sensors and the base station are all stationary after deployment.

3.1.2 Sensors are homogeneous and have the same capabilities. Each node is assigned a unique Identifier (ID).

3.1.3 Every node is assumed to use the same, fixed power level for intra-cluster communication (e.g. broadcasting and communicate with $\mathrm{CH}$ ). For the outside cluster communication, CHs are capable of increasing its transmission power level to reach its gateway node. And the gateway nodes can also use power control to vary the amount of transmission power according to the distance to the desired recipient [14].

3.1.4 Links are symmetric. A node can compute the approximate distance to another node based on the received signal strength, if the transmitting power is known.

\subsection{MCR Algorithm}

The concrete steps of MCR protocol are as below:

3.2.1 Build up the network topology:

This stage is comprised of three sub-stages:

3.2.1.1 Gateway nodes selection:

Firstly, the sink node adjusts its transmission power and broadcasts SinkMesg message to the sensor area. A node which receives this broadcast sends Child Re $q$ message to the sink node applying for being gateway node. Then the node processes in wait state. A node receiving the reply message 
from Sink node indicates that it has been a gateway node then goes to sleep.

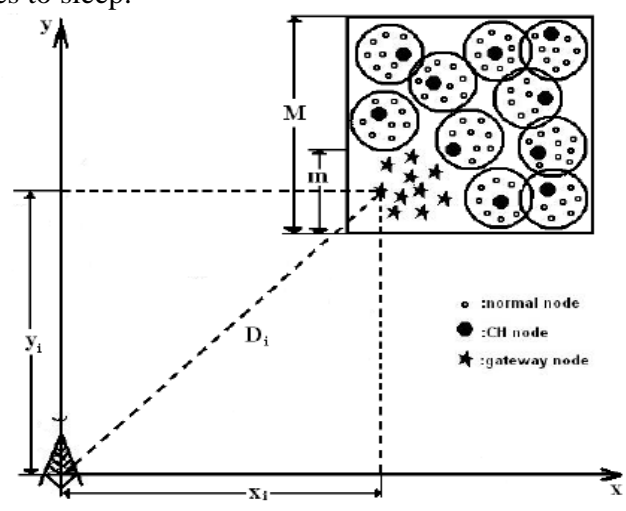

Fig. 2 Network topology of Multi-hop Clustering

\subsubsection{Clustering process:}

In this stage, the rest of the nodes except the gateways execute HEED to construct the clusters. That is to say, we use HEED cluster as the underlying clustering approach for MCR because of its generality and energy efficiency. After completion, all the normal nodes go to sleep whereas the $\mathrm{CH}$ nodes and gateway nodes go into the third phase.

\subsubsection{Each CH node connects its gateway node:}

In order to balance the energy consumption well and avoid the gateway nodes being failure too quickly. This protocol limits that a gateway node can connect with only one $\mathrm{CH}$ node. Initially, each gateway node adjusts its transmission power and broadcasts its initial message. A node which receives this broadcast, sets up a temporary father-nodes form Temp Father node Form first, records the values of the gateway node's ID, LEVEL and RSSI. If the number of the temporary fathernodes in a node's Temp Father node Form is more than one, it sorts the form according to the value of RSSI and picks up the maximal one as its final father node, then sends apply message to it. After receiving reply message, a node indicates that it has been in net. So far, the network topology has been built up, and the network topology is shown in Fig. 2. All nodes move into the Data collection and transmission stage.

3.2.1.4 Data collection and transmission:

In this stage, cluster member nodes collect data and transmit to their cluster-head nodes. Each cluster-head fuse the data receiving from all its cluster members and then send to its gateway node. The task for the gateway node is to send data to the sink node. Each node will check it's remain energy after accomplish its transmission assignment.

\subsection{Cluster-head rotation and network topology rebuild}

\subsubsection{Cluster-head rotation}

In this stage MCR has improved HEED. In HEED, clusterhead rotation is driven by time; this means that cluster-head rotation is triggered periodically. However, MCR adopts energy-driven method to rotate cluster-head. The purpose of this improvement is to reduce the extra energy consumption made by the time-driven cluster-head rotation. In MCR, each cluster-head applies for rotation according to its remaining energy and the action is only within its own cluster. That can not only avoid disturbing the normal operation of other clusters with high remain energy, but also can balance the energy consumption well in the network.

\subsubsection{Network topology rebuild}

MCR requires that all nodes move into the network topology rebuild stage when every four nodes lose their effectiveness. That is to say, when there has four new dead nodes in the network, all the live nodes execute the three stages above again.

Table1

Transmitter Parameters

\begin{tabular}{|c|c|}
\hline Initial energy & $12.1 \mathrm{~J}$ \\
\hline Mac type & IEEE802.11 \\
\hline Routing type & MCR \\
\hline Transmitter power & $0.305 \mathrm{~J}$ \\
\hline Receiver power & $0.395 \mathrm{~J}$ \\
\hline Ideal power & $0.335 \mathrm{~J}$ \\
\hline Transmission rate & $450 \mathrm{kbps}$ \\
\hline
\end{tabular}

\section{SIMULATION RESULTS}

All simulations have been implemented using NS2 simulator.

\subsection{Sources Vs Energy}

The above graph shows that the MCR Energy consumption is very low compared to HEED by varying load.

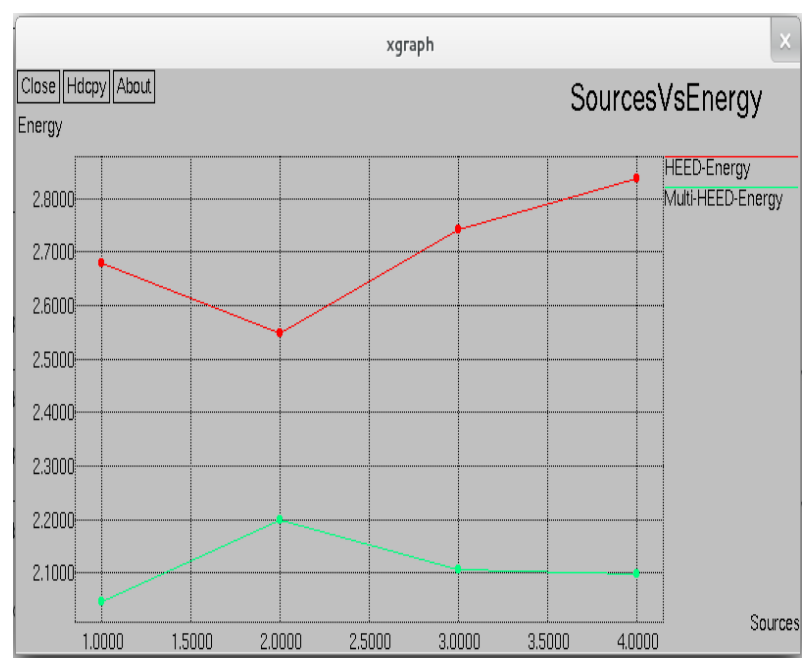

Fig. 4.1 Snapshot of Sources VS Energy

\subsection{Sources vs Del-Ratio:}

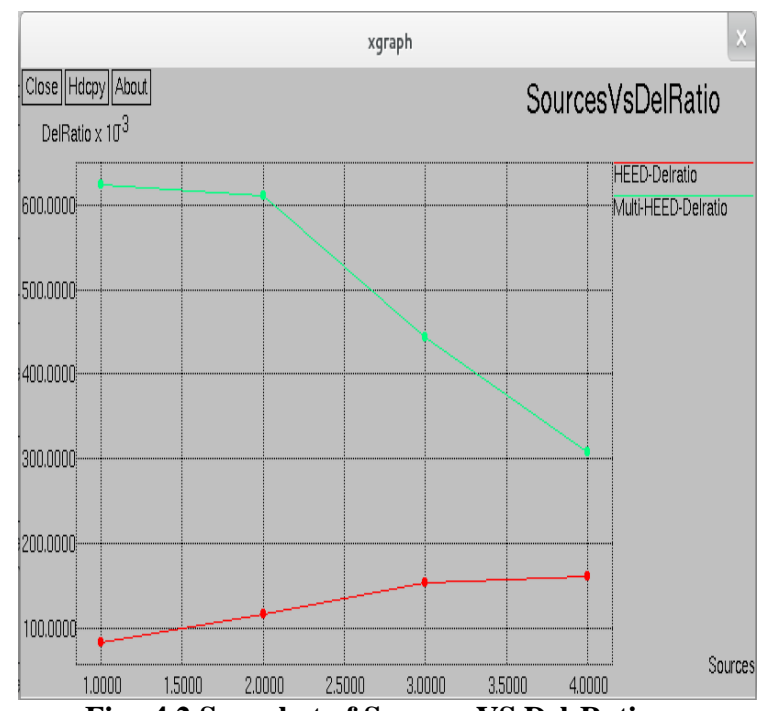

Fig. 4.2 Snapshot of Sources VS Del-Ratio 
The above graph shows that the MCR throughput is very high compare to HEED with respect to the number of sources.

\subsection{Sources Vs Packet Drop}

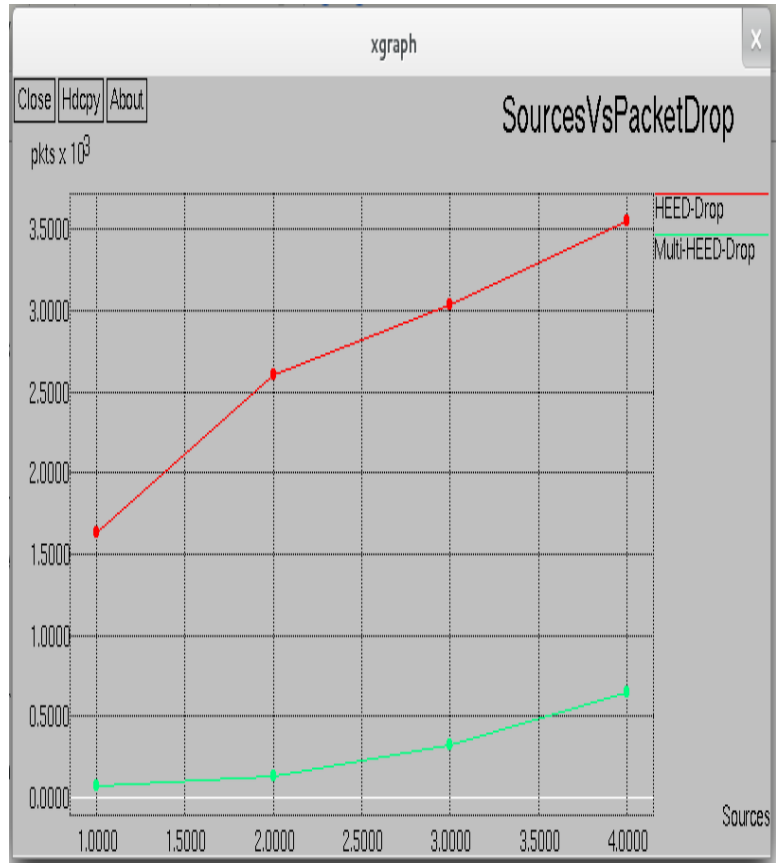

Fig. 4.3 Snapshot of Sources VS Packet Drop

The above graph shows that the MCR Packet drop is very low compare to HEED with respect to the number of sources.

\subsection{Rate Vs Energy}

xgraph

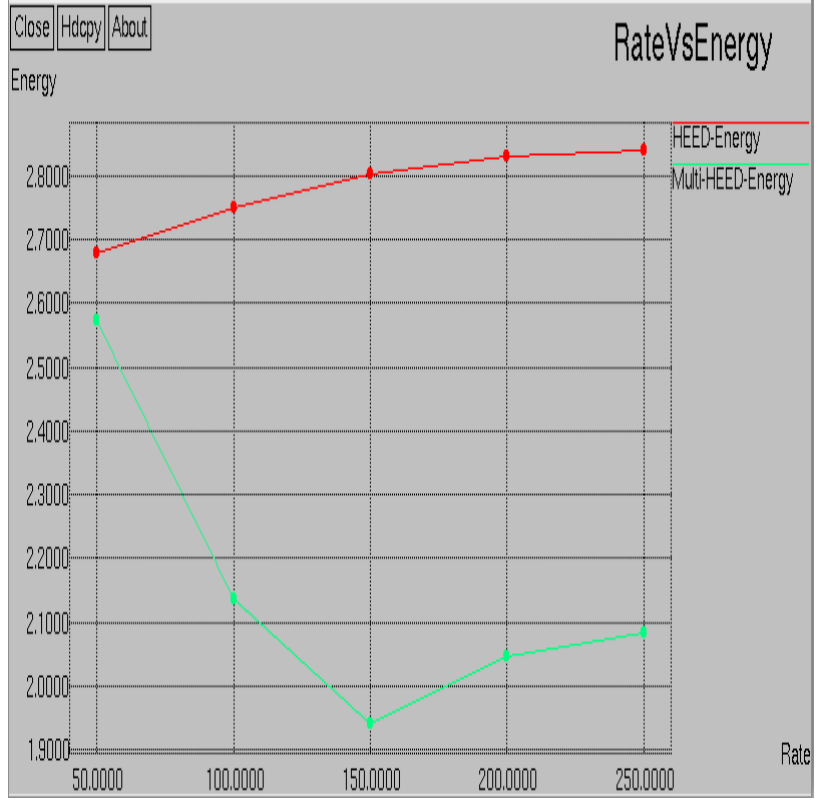

Fig. 4.4 Snapshot of Rate Vs Energy

The above graph shows that the MCR Energy consumption is very low compare to HEED with respect to the rate.

\subsection{Rate Vs Delratio}

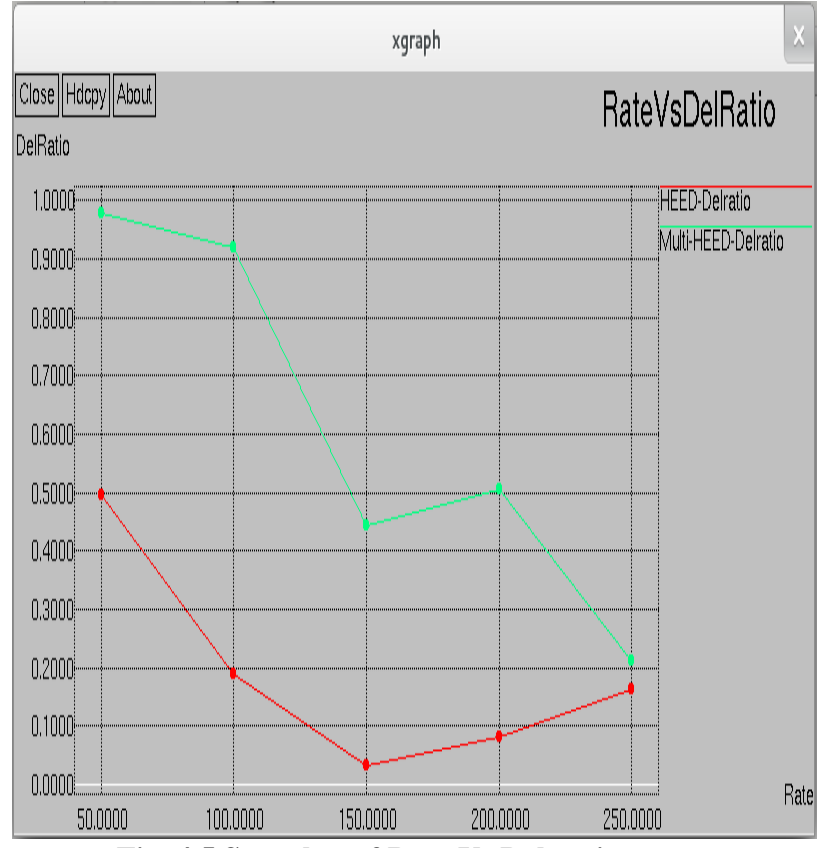

Fig. 4.5 Snapshot of Rate Vs Del-ratio

The above graph shows that the MCR throughput is very high compare to HEED with respect to the Rate.

\subsection{Rate Vs Packet Drop}

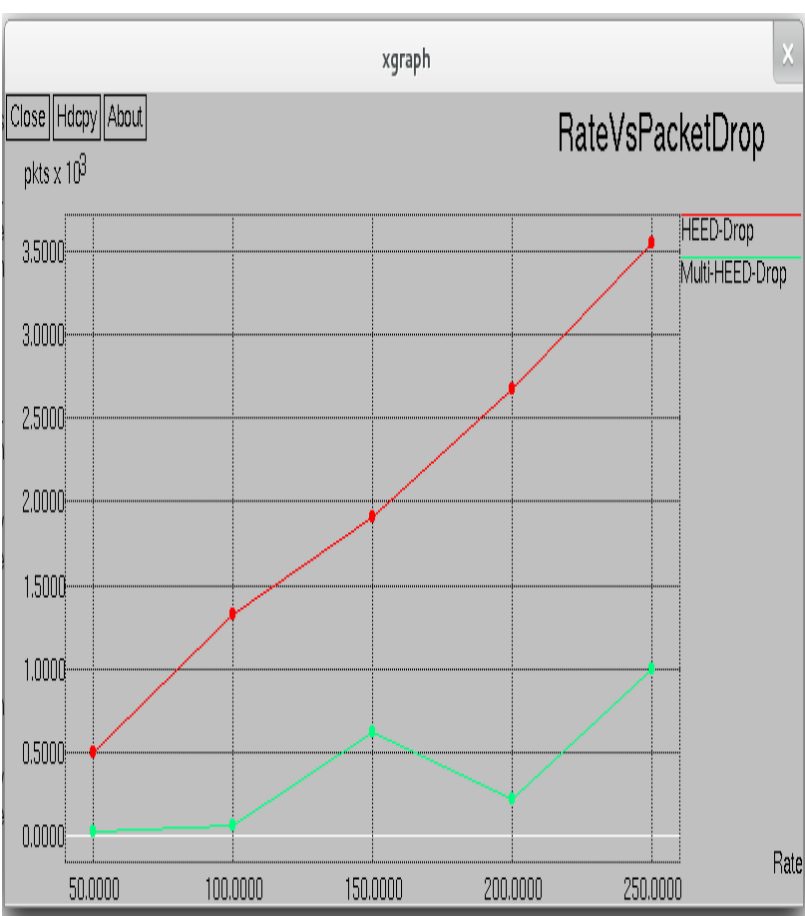

Fig 4.6 Snapshot of Rate Vs Packet Drop

The above graph shows that the MCR Packet drop is very low compare to HEED with respect to Rate. 


\section{CONCLUSION}

In this paper, a Multi-hop Clustering Routing Protocol (MCR) for long range transmission in wireless sensor network is proposed. Gateway nodes is used to forward from the $\mathrm{CHs}$ to the base station, so that the $\mathrm{CHs}$ can preserve some energy in data transmission and the gateway nodes can lighten their burden by not participating in clustering. Furthermore, an energy-driven method to rotate cluster-head instead of timedriven cluster-head rotation is also adopted to balance the energy consumption. Simulation results show that MCR obviously increases the network lifetime and well balances the energy consumption among the sensor nodes using NS2.

\section{REFERENCES}

[1] F. Akyildiz, W. Su, et al, "A survey on sensor networks", Communications Magazine, IEEE, vol. 40, no. 8, pp.102-114, 2002.

[2] H. W. Kim, H. S. Seo, et al, "Modeling of Energyefficient Applicable Routing Algorithm in WSN", International Journal of Digital Content Technology and its Applications, vol. 4, no. 5, pp. 13-22, 2010.

[3] [W. B. Heinzelman, A. P. Cnandrakasan, et al, "An application-specific protocol architecture for wireless microsensor networks," IEEE Transactions on Wireless Communications, vol. 1, no. 4, pp.660-670, 2002.

[4] Wesam Almobaideen, Khaled Hushaidan, Azzam Sleit, Mohammad Qatawneh , "A Cluster-Based Approach for Supporting QoS in Mobile Ad Hoc Networks ", International Journal of Digital Content Technology and its Applications, Vol. 5, No.1, pp. 1-9, 2011

[5] W. R. Heinzelman, A. Chandrakasan, et al, "EnergyEfficient Communication Protocol for Wireless Microsensor Networks," In Proceeding of the 33rd Hawaii International Conference on System Sciences, pp.1-10, 2000.

[6] O. Younis, S. Fahmy, "HEED: A hybrid, energyefficient, distributed clustering approach for ad hoc sensor networks," IEEE Transactions on Mobile Computing, vol. 3, no. 4, pp.366-379, 2004.

[7] F. Li, G. H. Chen, et al, "An Uneven Cluster-Based Routing Protocol for Wireless Sensor Networks," Chinese Journal of Computers. vol.30, no. 1, pp.27-36, 2007.

[8] J. Ibriq, I. Mahgoub, "Cluster-Based Routing in Wireless Sensor Networks: Issues and Challenges," Proc. of the 2004 Symposium on Performance Evaluation of Computer Telecommunication Systems, pp.759-766, 2004.
[9] P. Tillapart, S. Thammarojsakul, et al, "An Approach to Hybrid Clustering and Routing in Wireless Sensor Networks," Proceedings of the IEEEAC, Big Sky, MT, pp.1-8, 2005.

[10] R. S. Chang, C. J. Kuo, "An Energy Efficient Routing Mechanism for Wireless Sensor Networks," Proceedings of the 20th International Conference on Advanced Information Networking and Applications, Vienna, Austria, pp.308-312, 2006.

[11] KeyKhosravi, A. Ghaffari, et al, "New clustering protocol to decrease probability failure nodes and increasing the lifetime in WSNs," International Journal of Advancements in Computing Technology, vol. 2, no. 2, pp.117-121, 2010

[12] NS2, http://www.nsnam.org

[13] G. H. Chen, C. F. Li, et al, "An unequal cluster-based routing protocol in wireless sensor networks," Wireless Networks, vol. 15, no. 2, pp.193-207, 2009. - 325.

[14] "An Multi-hop Cluster Based Routing Protocol for Wireless Sensor Networks", I Yang, Yuxiang Zhuang, Hui Li,-Journal of Convergence Information Technology, Volume 6, Number 3. March 2011.

[15] http://opnet.com/simulator

\section{AUTHOR'S PROFILE}

S.Koteswararao graduated in Electronics Communication Engineering from Dr.S.G.I.E.T and post graduated in instrumentation and Control Systems from JNTUK, Kakinada and pursuing his $\mathrm{PhD}$ from JNTUK, Kakinada and research interests includes MAC Layer issues of Wireless sensor networks and Digital communication.

Dr.M.Sailaja obtained B.E in Electronics \&CommunicationEngineering. She has completed M.S in USA and $\mathrm{PhD}$ from JNTU hyd. presently; she is working as professor in ECE Department in JNTU Kakinada. Her Research interests include Parallel Processing and Computer Networks.

Dr. Tenneti Madhu obtained his B.E.degree from University of Madras, M.Tech from REC, Kurukshetra in 1994 and PhD from Osmania University in 2004.His research interests include GPS Data Analysis, Image Processing,Nano Technology and VLSI design and also published more no. of good journals. 